Утверждение 5. Пусть $g(x+2 y)=a(x, y)+2 b(x, y)$, где $x, y \in \mathbb{Z}_{2}^{n}$ и $a, b-$ булевы функции от $2 n$ переменных, является бент-функцией. Тогда функция $g^{\prime}(x+2 y)=$ $=3 a(x, y)+2 b(x, y)$ также является кватернарной бент-функцией от $n \geqslant 1$ переменных.

Отметим, что утверждение верно и в обратную сторону.

\title{
ЛИТЕРАТУРА
}

1. Matsui M. Linear cryptanalysis method for DES cipher // Eurocrypt'1993. LNCS. 1994. V. 765. P. 386-397.

2. Adams C. Constructing symmetric ciphers using the CAST design procedure // Design, Codes, and Cryptography. 1997. V.12. No. 3. P. 283-316.

3. Hell M., Johansson T., Maximov A., and Meier W. A stream cipher proposal: Grain-128// IEEE Intern. Symp. Inform. Theory. Seattle, WA, 2006. P. 1614-1618.

4. Tokareva N. Bent Functions: Results and Applications to Cryptography. Acad. Press, Elsevier, 2015. $230 \mathrm{p}$.

5. Kumar P. V., Scholtz R. A., and Welch L. R. Generalized bent functions and their properties // J. Combin. Theory. 1985. V.40. No. 1. P. 90-107.

6. Solé P. and Tokareva N. Connections Between Quaternary and Binary Bent Functions // Cryptology ePrint Archive, Report 2009/544. http://eprint.iacr.org/.

7. Solé P. and Tokareva $N$. On quaternary and binary bent functions // Прикладная дискретная математика. Приложение. 2009. №1. С. 16-18.

\section{ON A SECONDARY CONSTRUCTION OF QUADRATIC APN FUNCTIONS}

\begin{abstract}
K. V. Kalgin, V. A. Idrisova
Almost perfect nonlinear functions possess the optimal resistance to the differential cryptanalysis and are widely studied. Most known constructions of APN functions are obtained as functions over finite fields $\mathbb{F}_{2^{n}}$ and very little is known about combinatorial constructions in $\mathbb{F}_{2}^{n}$. We consider how to obtain a quadratic APN function in $n+1$ variables from a given quadratic APN function in $n$ variables using special restrictions on new terms.
\end{abstract}

Keywords: vectorial Boolean function, APN function, quadratic function, secondary construction.

Let us recall some definitions. Let $\mathbb{F}_{2}^{n}$ be the $n$-dimensional vector space over $\mathbb{F}_{2}$. A function $F$ from $\mathbb{F}_{2}^{n}$ to $\mathbb{F}_{2}^{m}$, where $n$ and $m$ are integers, is called a vectorial Boolean function. If $m=1$, such a function is called Boolean. Every vectorial Boolean function $F$ can be represented as a set of $m$ coordinate functions $F=\left(f_{1}, \ldots, f_{m}\right)$, where $f_{i}$ is a Boolean function in $n$ variables. Any vectorial function $F$ can be represented uniquely in its algebraic normal form (ANF):

$$
F(x)=\sum_{I \in \mathcal{P}(N)} a_{I}\left(\prod_{i \in I} x_{i}\right),
$$

where $\mathcal{P}(N)$ is a power set of $N=\{1, \ldots, n\}$ and $a_{I} \in \mathbb{F}_{2}^{m}$. The algebraic degree of a given function $F$ is the degree of its $\mathrm{ANF}: \operatorname{deg}(F)=\max \left\{|I|: a_{I} \neq 0, I \in \mathcal{P}(N)\right\}$. If algebraic

\footnotetext{
${ }^{1}$ The work was carried out within the framework of the state contract of the Sobolev Institute of Mathematics (project no. 0314-2019-0017) and supported by RFBR (projects no. 18-07-01394, 20-31-70043) and Laboratory of Cryptography JetBrains Research.
} 
degree of a function $F$ is not more than 1 , then $F$ is called affine. If for an affine function $F$ it holds $F(\mathbf{0})=\mathbf{0}$, then $F$ is called linear. If algebraic degree of a function $F$ is equal to 2, then $F$ is called quadratic. Two vectorial functions $F$ and $G$ are extended affinely equivalent (EA-equivalent) if $F=A_{1} \circ G \circ A_{2}+A$, where $A_{1}, A_{2}$ are affine permutations on $\mathbb{F}_{2}^{n}$ and $A$ is an affine function. Let $F$ be a vectorial Boolean function from $\mathbb{F}_{2}^{n}$ to $\mathbb{F}_{2}^{n}$. For vectors $a, b \in \mathbb{F}_{2}^{n}$, where $a \neq 0$, consider the value

$$
\delta(a, b)=\left|\left\{x \in \mathbb{F}_{2}^{n}: F(x+a)+F(x)=b\right\}\right| .
$$

Denote by $\Delta_{F}$ the following value:

$$
\Delta_{F}=\max _{a \neq \mathbf{0}, b \in \mathbb{F}_{2}^{n}} \delta(a, b)
$$

Then $F$ is called differentially $\Delta_{F}$-uniform function. The smaller the parameter $\Delta_{F}$ is, the better the resistance of a cipher containing $F$ as an $S$-box to differential cryptanalysis. For the vectorial functions from $\mathbb{F}_{2}^{n}$ to $\mathbb{F}_{2}^{n}$, the minimal possible value of $\Delta_{F}$ is equal to 2 . In this case, the function $F$ is called almost perfect nonlinear $(A P N)$. This notion was introduced by K. Nyberg in [1]. APN fuctions draw attention of many researchers, but there is still a significant list (see, for example, [2-4]) of important open questions. We are especially interested how to find new constructions of APN functions in vector space $\mathbb{F}_{2}^{n}$, since almost all the known constructions of this class are found only as polynomials over the finite fields, and to the best of our knowledge, only a few approaches to such combinatorial constructions was proposed [5, 6].

Since EA-equivalence preserves APNness, it is always possible to omit linear and constant terms in the algebraic normal form of a given APN function. Further we will consider quadratic vectorial Boolean functions that have only quadratic terms in their ANF. The following theorem gives a necessary condition on the ANF of a given APN function.

Theorem 1 [7]. Let $F=\left(f_{1}, \ldots, f_{n}\right)$ be an APN function in $n$ variables. Then every quadratic term $x_{i} x_{j}$, where $i \neq j$, appears at least in one coordinate function of $F$.

This property motivated us to suggest the following construction of quadratic APN functions. Let $G=\left(g_{1}, \ldots, g_{n}\right)$ be a quadratic APN-function in $n$ variables. Consider vectorial function $F=\left(f_{1}, \ldots, f_{n}, f_{n+1}\right)$ in $n+1$ variables such that

$$
\begin{gathered}
f_{1}=g_{1}+\sum_{i=1}^{n} \alpha_{1, i} x_{i} x_{n+1}, \\
\ldots \\
f_{n}=g_{n}+\sum_{i=1}^{n} \alpha_{n, i} x_{i} x_{n+1}, \\
f_{n+1}=g_{n+1}+\sum_{i=1}^{n} \alpha_{n+1, i} x_{i} x_{n+1},
\end{gathered}
$$

where $\alpha_{1, i} \ldots, \alpha_{n+1, i} \in \mathbb{F}_{2}$ for $i=1, \ldots, n$ and $g_{n+1}=\sum_{1 \leqslant j<k \leqslant n} \beta_{j, k} x_{j} x_{k}$ for some fixed $\beta_{j, k} \in \mathbb{F}_{2}$. Note that if $\alpha_{1, i}, \ldots, \alpha_{n, i}$ are such that each term $x_{i} x_{n+1}$ appears at least in one of the coordinate functions $f_{1}, \ldots, f_{n}$, then the necessary condition of Theorem 1 is held for the constructed function $F$.

Each quadratic vectorial function $G$ in $n$ variables can be considered as a symmetric matrix $\mathcal{G}=\left(g_{i j}\right)$, where each element $g_{i j} \in \mathbb{F}_{2}^{n}$ is a vector of coefficients corresponding to 
term $x_{i} x_{j}$ in the algebraic normal form of $G$ and all diagonal elements $g_{i i}$ are null. It is necessary to mention that these matrices are essentially the same as so-called QAM matrices that were used in $[8,9]$ to construct and classify a lot of new quadratic APN functions over finite fields. Using these matrices, the APN property can be formulated in the following way:

Proposition 1. Let $\mathcal{G}$ be the matrix that corresponds to quadratic vectorial function $G$. Then function $G$ is $\mathrm{APN}$ if and only if $x(\mathcal{G} \cdot a) \neq 0$ for all $x \neq a$, where $a, x \in \mathbb{F}_{2}^{n}$ and $a \neq \mathbf{0}$.

In terms of matrices, the construction (1) can be considered as an extension of a given $\mathcal{G}$ with an extra bit that represents $g_{n+1}$ in every element and an extra pair of row and column that represents a set of new terms $x_{i} x_{n+1}$.

Consider a quadratic APN function $G$ and the corresponding $n \times n$ matrix $\mathcal{G}$. Denote the vector of nonzero coefficients as $\alpha=\left(\alpha_{1}, \ldots, \alpha_{n}\right)$. Let us fix $g_{n+1}$ and construct $(n+1) \times$ $\times(n+1)$ matrix $\mathcal{F}$ by adding $\left(\alpha_{1}, \ldots, \alpha_{n}, 0\right)$ as the last column and the last row and adding new bit to every element according to the choice of $g_{n+1}$. Let us denote as $\mathcal{G}^{\prime}$ the submatrix $\left(f_{i j}\right)$ of $\mathcal{F}$, such that $i, j<n+1$. Let $\langle X\rangle$ denote the linear span of $X$ and $F$ be the quadratic vectorial function corresponding to the constructed matrix $\mathcal{F}$.

Theorem 2. A function $F$ is APN if and only if $\alpha \cdot a^{\prime}$ does not belong to $\left\langle\mathcal{G}^{\prime} \cdot a^{\prime}\right\rangle$ for all $a^{\prime} \in \mathbb{F}_{2}^{n}, a^{\prime} \neq \mathbf{0}$.

Theorem 2 shows how to choose new coefficients $\alpha_{1, i} \ldots, \alpha_{n+1, i} \in \mathbb{F}_{2}$ in the construction (1) such that an obtained function $F$ is APN. When $n=3,4$ and 5 , for APN functions that are representatives of EA classes, all possible classes of quadratic APNs are obtained for 4,5 and 6 variables from the classification [10] and large variety of classes for constructing functions in 6 and 7 variables.

\section{REFERENCES}

1. Nyberg K. Differentially uniform mappings for cryptography. EUROCRYPT'93, LNCS, 1994, vol. 765 , pp. 55-64.

2. Carlet C. Open questions on nonlinearity and on APN Functions. WAIFI 2014, LNCS, 2015, vol. 9061, pp. 83-107.

3. Glukhov M. M. O priblizhenii diskretnykh funktsiy lineynymi funktsiyami [On the approximation of discrete functions by linear functions]. Matematicheskie Voprosy Kriptografii, 2016, vol. 7, no. 4, pp. 29-50. (in Russian)

4. Tuzhilin M.E. Pochti sovershennye nelineynye funktsii [APN-functions]. Prikladnaya Diskretnaya Matematika, 2009, no. 3(5), pp. 14-20. (in Russian)

5. Gorodilova A.A. Characterization of almost perfect nonlinear functions in terms of subfunctions. Discrete Math. Appl., 2016, vol. 26, iss. 4, pp. 193-202.

6. Idrisova V.A. On an algorithm generating 2-to-1 APN functions and its applications to "the big APN problem". Cryptogr. Commun., 2019, no. 11, pp. 21-39.

7. Beth T. and Ding C. On almost perfect nonlinear permutations. EUROCRYPT'93, LNCS, 1993, vol. 765, pp. 65-76.

8. Yu Y., Wang M., and Li Y. A matrix approach for constructing quadratic APN functions. Des. Codes Cryptogr., 2014, no. 73, pp. 587-600.

9. Yu Y., Kaleyski N.S., Budaghyan L., and Li Y. Classification of Quadratic APN Functions with Coefficients in GF(2) for Dimensions up to 9. IACR Cryptol. ePrint Arch.: 1491, 2019.

10. Brinkmann $M$. and Leander $G$. On the classification of APN functions up to dimension five. Des. Codes Cryptogr., 2008, vol. 49, iss. 1-3, pp. 273-288. 